\title{
Open
}

\section{A synbiotic-containing amino-acid-based formula improves gut microbiota in non-IgE-mediated allergic infants}

\author{
David C.A. Candy ${ }^{1}$, Marleen T.J. Van Ampting ${ }^{2}$, Manon M. Oude Nijhuis ${ }^{2}$, Harm Wopereis ${ }^{3}$, Assad M. Butt ${ }^{1}$, Diego G. Peroni ${ }^{4}$, \\ Yvan Vandenplas ${ }^{5}$, Adam T. Fox ${ }^{6}$, Neil Shah ${ }^{7}$, Christina E. West ${ }^{8}$, Johan Garssen ${ }^{9}$, Lucien F. Harthoorn ${ }^{2}$, Jan Knol $^{2}$ and \\ Louise J. Michaelis ${ }^{10}$; on behalf of the ASSIGN study group ${ }^{11}$
}

BACKGROUND: Prebiotics and probiotics (synbiotics) can modify gut microbiota and have potential in allergy management when combined with amino-acid-based formula (AAF) for infants with cow's milk allergy (CMA).

METHODS: This multicenter, double-blind, randomized controlled trial investigated the effects of an AAF-including synbiotic blend on percentages of bifidobacteria and Eubacterium rectale/Clostridium coccoides group (ER/CC) in feces from infants with suspected non-lgE-mediated CMA. Feces from age-matched healthy breastfed infants were used as reference (healthy breastfed reference (HBR)) for primary outcomes. The CMA subjects were randomized and received test or control formula for 8 weeks. Test formula was a hypoallergenic, nutritionally complete AAF including a prebiotic blend of fructo-oligosaccharides and the probiotic strain Bifidobacterium breve M-16V. Control formula was AAF without synbiotics.

RESULTS: A total of 35 (test) and 36 (control) subjects were randomized; HBR included 51 infants. At week 8, the median percentage of bifidobacteria was higher in the test group than in the control group (35.4\% vs. 9.7\%, respectively; $P<0.001)$, whereas ER/CC was lower (9.5\% vs. $24.2 \%$, respectively; $P<0.001)$. HBR levels of bifidobacteria and ER/CC were $55 \%$ and $6.5 \%$, respectively.

CONCLUSION: AAF including specific synbiotics, which results in levels of bifidobacteria and ER/CC approximating levels in the HBR group, improves the fecal microbiota of infants with suspected non-IgE-mediated CMA.

C ow's milk allergy (CMA) affects up to $5 \%$ of infants and children in Western countries (1-3), although the number of challenge-proven cases may be lower (4). CMA is associated with a range of distressing and potentially severe clinical symptoms affecting the skin, gastrointestinal (GI) tract, and, less commonly, the respiratory tract (5). Infants with non-IgE CMA generally have delayed symptoms (6) and present particular challenges because of difficulty in diagnosis, lack of validated tests, and a paucity of clinical studies (7). When exclusive breastfeeding is not possible, or there is failure of control of symptoms when dairy ( \pm soya) is eliminated out of the maternal diet, the dietary management guidelines for infants with CMA recommend extensively hydrolyzed formula (eHF) for mild cases and amino-acidbased formula (AAF) for severe cases. When eHF is not tolerated and fails to resolve allergy symptoms, AAF is recommended $(6,8)$.

Aberrant composition and diversity of gut microbiota in early life may disrupt development of the immune system (9$11)$, which is associated with allergy-related diseases (12-14), including food allergies, such as CMA (15). A study of infants with CMA showed that gut microbiota composition at 36 months was associated with allergy resolution by the age of 8 years (16), suggesting that, during infancy, gut microbiota could be a potential mechanism to influence food allergy outcomes in childhood.

Studies showing that prebiotics and probiotics, or their combination (synbiotics), can positively modulate the composition of gut microbiota (17-21), provided the rationale to investigate such an approach in CMA. In addition, clinical studies of eHF supplemented with probiotics showed improved symptoms in infants with CMA (22-25); however, eHF may not be the most appropriate formula for patients with non-IgE CMA, such as those presenting with faltering growth or those with persistent clinical symptoms when using eHF, (6) and an AAF is recommended in such cases (8). Clinical studies in healthy infants (26) and infants with CMA $(26,27)$ showed that synbiotic-supplemented AAF was hypoallergenic, well tolerated, and supported normal growth $(26,27)$. On the basis of these findings, we conducted a randomized trial to assess the effect of an AAF with a specific

\footnotetext{
${ }^{1}$ Royal Alexandra Children's Hospital, Brighton, UK; ${ }^{2}$ Nutricia Research, Nutricia Advanced Medical Nutrition, Utrecht, The Netherlands; ${ }^{3}$ Laboratory of Microbiology, Wageningen University, Wageningen, The Netherlands; ${ }^{4}$ University Hospital Verona, Verona, Italy; ${ }^{5}$ University Hospital Brussels, Brussels, Belgium; ${ }^{6}$ Guy's and St Thomas' Hospitals NHS Foundation Trust, London, UK; ${ }^{7}$ Great Ormond Street Hospital, London, UK; ${ }^{8}$ Umeå University, Umeå, Sweden; ${ }^{9}$ Utrecht Institute for Pharmaceutical Sciences, Faculty of Science, Utrecht University, Utrecht, The Netherlands; ${ }^{10}$ Great North Children's Hospital, Newcastle upon Tyne, UK. Correspondence: Marleen T.J. Van Ampting (marleen.vanampting@nutricia.com)

${ }^{11} \mathrm{ASSIGN}$ investigators to this study group are listed before References.

Received 14 February 2017; accepted 7 October 2017; advance online publication 6 December 2017. doi:10.1038/pr.2017.270
} 
Table 1. Inclusion and exclusion criteria

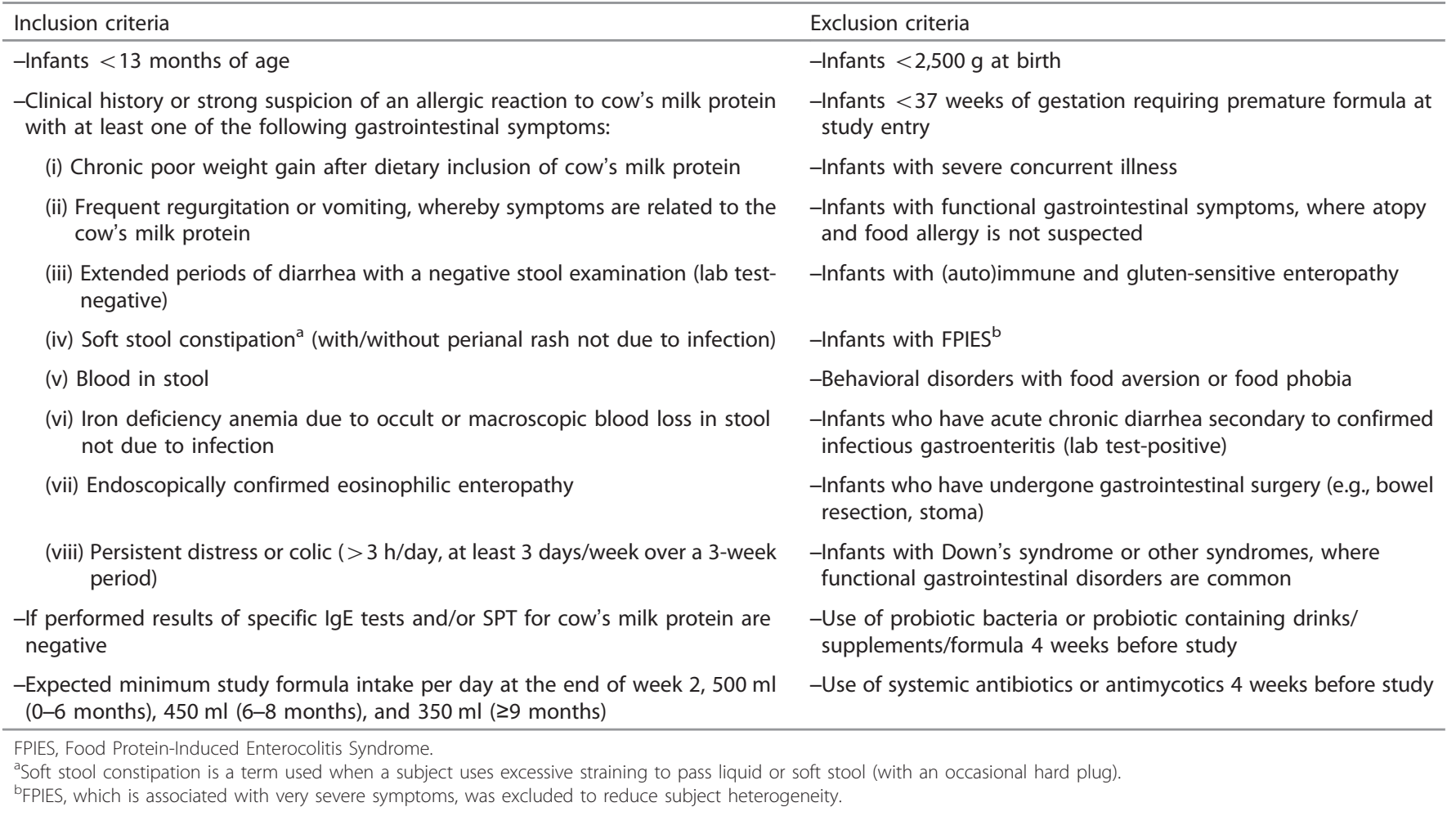

and optimized synbiotic blend on fecal microbiota composition and to explore clinical effectiveness in infants with suspected GI non-IgE-mediated CMA (28). This is the first randomized trial of a synbiotic-supplemented AAF exclusively in infants with suspected non-IgE CMA and includes an age-matched healthy breastfed reference group (HBR).

\section{METHODS}

\section{Trial Design}

This was a multicenter, double-blind, randomized controlled trial (Netherlands Trial Register NTR3979) including subjects with suspected non-IgE CMA and a non-randomized reference group (HBR). Subjects were recruited by Great North Children's Hospital, Newcastle; Barts/Royal Hospital, Guys and St Thomas' Hospital, GOSH, London; Royal Alexandra Childrens Hospital, Brighton in the UK; University Hospital Padova, Padua; University Hospital Verona, Verona in Italy; CU St Luc, University Hospital Brussels, Huderf, Brussels in Belgium; and Umeå University, Umeå, Sweden. Eligible subjects, enrolled from 1 October 2013 to 30 April 2015, were stratified based on predominant, investigator-assessed symptoms (skin or GI) and randomly allocated to either test or control formula. The random allocation, by using a central Interactive Web Response System (Orca Pharma, Heesch, The Netherlands), was performed by a generated sequence/algorithm using block randomization to ensure that the test and control formulae were assigned equally. Formulae were identically packaged in $400 \mathrm{~g}$ tins and labeled with a one-letter code so that parents/guardians and those assessing outcomes were blind to the group assignment.

Test and control formulae were given for 8 weeks, after which the subjects were switched to a prescribed formula appropriate for their condition and age according to the local clinician's choice and practice. Cow's milk protein was introduced depending on local clinical practice. The subjects continued test or control formula if an AAF was considered the most appropriate approach for dietary management of clinical symptoms. Solids introduced in diets of the subjects were recorded by means of food diaries. The trial duration from screening to completion was a maximum of 28 weeks.

The trial was approved by the ethics committees of participating centers and all parents/guardians provided written informed consent.

\section{Participants}

Subjects were randomized if they were aged $<13$ months and had a clinical history or strong suspicion of an allergic reaction to cow's milk protein, based on a robust diagnostic work-up (Table 1) collectively designed by a multidisciplinary team of clinicians, comprising pediatric gastroenterology, allergy, and immunology specialists. The defined inclusion criteria (Table 1) included a negative specific IgE test (ImmunoCAP), and/or a negative skinprick test with cow's milk protein, if a test was performed (testing was not mandatory per protocol). In addition, at study entry, the subjects had at least one of the following (GI) symptoms related to inclusion of cow's milk protein in their diet: faltering growth; frequent regurgitation or vomiting; extended periods of diarrhea with a negative stool examination (negative microbiology and virology laboratory tests); soft stool constipation; blood in stool; irondeficiency anemia due to occult or macroscopic blood loss in stools not due to infection or dietary insufficiency; endoscopically confirmed eosinophilic enteropathy; or persistent distress or colic ( $>3 \mathrm{~h}$ per day at least 3 days per week over a 3 -week period). Infants were excluded for the following reasons: birth weight $<2,500 \mathrm{~g}$, $<37$ weeks' gestation requiring specific premature infant formula at study entry, severe concurrent illness, functional GI symptoms without suspicion of atopy and food allergy, immune, autoimmune, or gluten-sensitive enteropathy, food protein-induced enterocolitis syndrome, acute or chronic diarrhea secondary to a confirmed infectious gastroenteritis, behavioral disorders with food aversion or food phobia, GI surgery, syndromes commonly associated with functional GI disorders, and the use of probiotics, systemic antibiotics, or antimycotic drugs 4 weeks preceding study entry. 


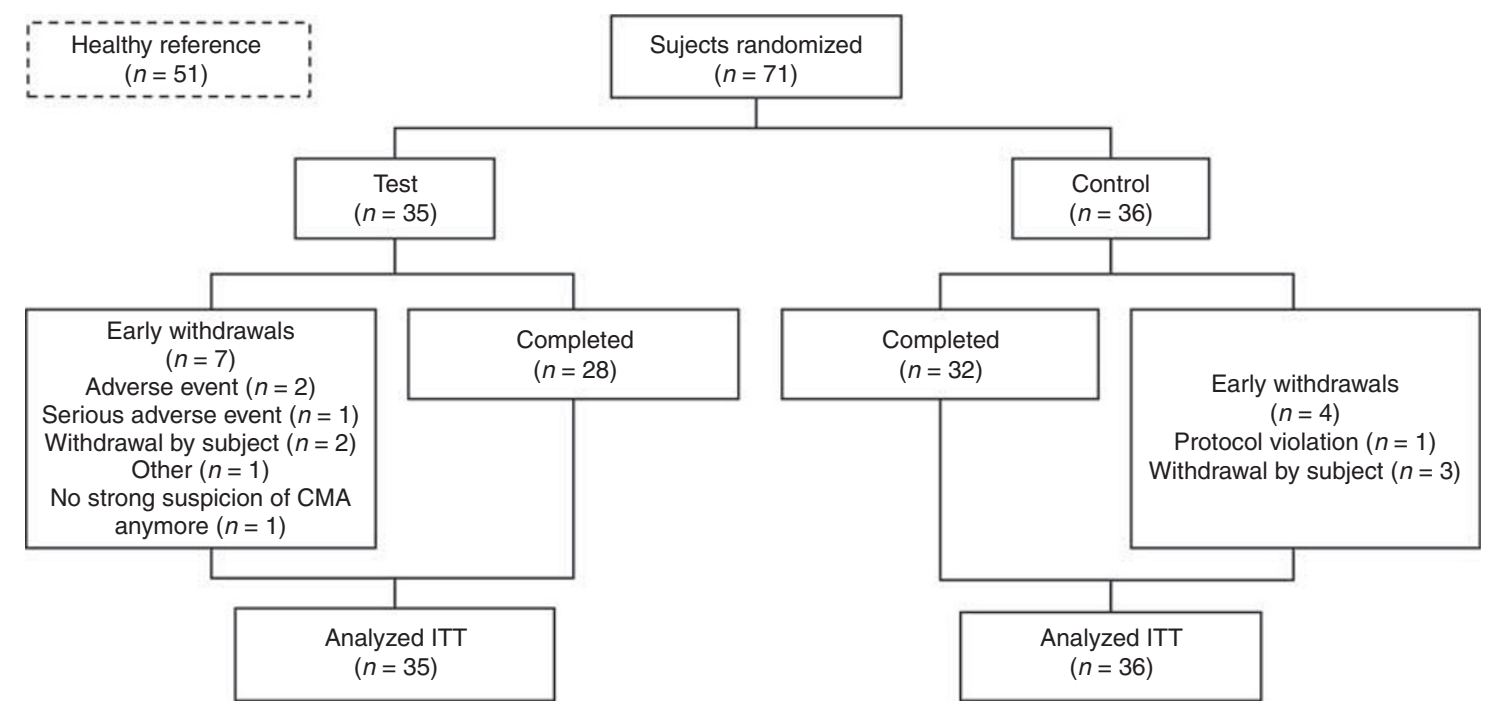

Figure 1. CONSORT diagram showing the flow of subjects in the randomized arms. IT, intent to treat. Early withdrawal-related adverse events were constipation $(n=1)$ and infantile colic $(n=1)$, and related serious adverse event $(n=1)$ was viral laryngitis. The events were reported as unlikely and not related to study formula.

Two weeks after randomization, symptom resolution was evaluated and subjects with persistent symptoms were reassessed by the investigator, and only subjects with suspicion of, or confirmed, non-IgE CMA continued in the study. Subjects not eligible at reassessment were withdrawn (Figure 1).

The non-randomized, HBR group comprised infants who were exclusively breastfed until 7 months of age. Healthy subjects of 7 months or older did consume solids, which were recorded in dietary diaries. In addition, the subjects did not have any concurrent illness or clinical history of allergy, did not receive any treatment or nutritional intervention, and were within a similar age range to subjects in the randomized groups. Infants in the HBR group were prospectively recruited from selected study centers and local community centers.

\section{Interventions in the Randomized Arms}

The test formula was a hypoallergenic, nutritionally complete AAF (Neocate LCP; Nutricia Advanced Medical Nutrition, Liverpool, UK) containing a prebiotic blend of chicory-derived neutral oligofructose and long-chain inulin (BENEO-Orafti SA, Oreye, Belgium; 9:1 ratio at a total concentration of $0.63 \mathrm{~g} / 100 \mathrm{ml}$ ) and a probiotic strain Bifidobacterium breve M-16 V (Morinaga Milk Industry, Tokyo, Japan) at a concentration of $1.47 \times 10^{9}$ colony-forming units (CFU)/ $100 \mathrm{ml}$ formula. The control formula was a commercially available AAF without synbiotics (Neocate LCP; Nutricia Advanced Medical Nutrition). Subjects were instructed to consume a minimum, agespecific, daily formula intake from the end of week 2 (infants aged 0-6 months, $500 \mathrm{ml}$; 6-8 months, $450 \mathrm{ml}$; and >9 months, $350 \mathrm{ml}$ ).

\section{Assessments}

Medical history was documented by the clinician, for both test and control group, at baseline (week 0) and via 24-h recall of baseline presenting complaints. Stool samples for analysis were collected by parents/guardians at week 0 , if possible under the supervision of a health-care professional, and then at home at weeks 8, 12, and 26. The samples collected into $10 \mathrm{ml}$ stool containers (Greiner Bio-One, Kremsmünster, Austria) were immediately frozen at $-80^{\circ} \mathrm{C}$ in the clinic or at $-20^{\circ} \mathrm{C}$ in a home freezer before transferring it to the clinic storage facility. Parents/guardians completed a diary to record stool characteristics (frequency, color, and consistency; over 3 days during weeks $1,4,8,12$, and 26), study formula intake (volume consumed over 7 days during weeks 1,4 , and 8), and diet evaluation (type of foods eaten by the subject at the end of each week during weeks 1, 4, 8, 12, and 26). Skin symptoms (including atopic dermatitis), respiratory symptoms (blocked nose, coughing, and wheezing), GI symptoms (vomiting, spitting up), and general symptoms (ease to settle or burp after feeds, and visual signs of discomfort, e.g., back arching and crying due to irritability) were recorded in the diary (collected over 3 days during weeks $1,4,8,12$, and 26) and reviewed by the investigator during clinic visits. In order to collect HBR stool samples that could be age-matched with the week 8 age range of the CMA infants, stool samples from HBR were collected at one time point, or more if feasible for infant and parents. The stool sample collection was as described above and accompanied with a completed diet diary and stool characteristic assessment. After study completion, and before deblinding the groups, age-matching HBR samples were selected for reference analyses.

\section{Objectives and Outcomes}

The primary objective was to assess the effect of test formula on fecal percentages of bifidobacteria and Eubacterium rectale/Clostridium coccoides group (ER/CC) at 8 weeks, determined by florescence in situ hybridization analysis using $16 \mathrm{~S}$ rRNA-targeted oligonucleotide probes, as described previously (29). Bifidobacteria are typically abundant in healthy breastfed infants (30) and show stable and increased levels in early childhood compared with adults (31). As maturation to adult-like profiles may extend beyond 5 years of age, $\mathrm{ER} / \mathrm{CC}$ was selected as a marker because it typically is one of the first adult-like bacterial groups appearing in the infant gut $(30,31)$.

Assessing the effects of test formula on stool characteristics at weeks 0 and 8 was a secondary objective.

Measuring clinical effectiveness of test formula on allergic symptoms was an exploratory objective. Skin symptoms at weeks 0 and 8 were evaluated by the SCORing Atopic Dermatitis (SCORAD) rating scale (32). Parent-reported rating scales for skin, respiratory, GI, and general symptoms were collected using a four-point scale, where a score of 1 was taken as normal with no symptoms. Assessed parentreported symptoms were evaluated together with the clinician during visits.

The frequency and severity of adverse events and use of concomitant medication were used to assess safety and tolerability. Standard anthropometric measurements were recorded to assess growth. 


\section{Statistics}

Sample size estimation was based on effect size difference of $26.4 \%$ in bifidobacteria and $23.6 \%$ in the ER/CC group (29). Assuming a significance level of $5 \%$ using a two-sided statistical test and Hochberg principle for two parameters, a sample size of 20 completers per study arm gave $80 \%$ power to observe an effect. Assuming estimated drop-out rates of up to $25 \%$ of subjects whose symptoms did not resolve within 2 weeks of starting AAF and $20 \%$ for other reasons, 68 subjects were to be recruited. Following a semiblinded interim analysis by an independent committee, it was decided to keep the sample size unchanged. This committee consisted of a clinical study expert, a gut microbiology expert, and a statistician. These experts were not involved in any discussion or decision regarding conduct of the study or study results after they evaluated semi-blinded data.

Overall statistical analyses were performed comparing test with the control group. To bring microbiota outcomes in a context of a healthy situation, levels of a reference group (HBR) were determined and used as reference only and not as a treatment group. Two primary outcome parameters (bifidobacteria and ER/CC) were recorded as percentages of total fecal bacteria. All analyses were performed on intention-to-treat data set (ITT), defined as all randomized subjects. Analysis of covariance (ANCOVA) was performed with a between-subjects factor "group" (test vs. control) and both the stratification factor (skin or GI) and the baseline measure as covariate (the primary model). The model-based intervention effect size was calculated, and the significance of the fixed parameter "group" estimate was evaluated. To evaluate potential influence on primary outcome, additional sensitivity analyses were performed for the predetermined covariates: age at baseline, mode of delivery, sex, antibiotic use during study, introduction of weaning foods, total duration of breastfeeding, intake of proton pump inhibitors or $\mathrm{H} 2$ antagonists, study site, and country. For sensitivity analysis 1 (including only age at baseline as covariate) the estimate was compared with the estimate of the primary model; for sensitivity analysis 2 (including age at baseline and one of the other covariates) the estimate was compared with the estimate of sensitivity analysis 1 .

Secondary and exploratory outcome parameters were reported descriptively. $P$ values for SCORAD (change from baseline), clinical symptoms, and stool frequency (levels at week 8) were based on the Van Elteren test comparing test and control groups accounting for the stratification factor (skin or GI symptoms). $P$ values for the mean daily formula intake were tested by $t$-test and the median daily intake by means of Wilcoxon sum-rank test.

Statistical analyses were performed by using SAS (SAS Enterprise Guide version 4.3 or higher) for Windows (SAS Institute, Cary, NC). Results are expressed as mean values \pm SD unless stated otherwise.

\section{RESULTS}

A total of 71 subjects with suspected non-IgE CMA were recruited from 11 centers in the UK, Italy, Belgium, and Sweden. Figure $\mathbf{1}$ summarizes the flow of patients in the randomized treatment arms; 35 subjects were randomized to test formula and 36 to control formula. Early withdrawalrelated adverse events were constipation $(n=1)$ and infantile colic $(n=1)$, and a related serious adverse event $(n=1)$ was viral laryngitis. The events were reported as unlikely and not related to study formula.

A total of 110 stool samples from 60 healthy subjects were collected, and 51 subjects were considered eligible following predefined criteria; subsequently, 51 stool samples from these subjects were selected as healthy reference samples by agematching with age of CMA subjects at week 8 of intervention (Table 2). Characteristics of subjects in the randomized
Table 2. Demographics of subjects with CMA and the healthy reference group

\begin{tabular}{lcccc}
\hline & $\begin{array}{c}\text { Test } \\
(N=35)\end{array}$ & $\begin{array}{c}\text { Control } \\
(N=36)\end{array}$ & $\begin{array}{c}\text { Total } \\
\text { CMA } \\
(N=71)\end{array}$ & $\begin{array}{c}\text { Healthy } \\
\text { subjects } \\
(N=51)\end{array}$ \\
\hline $\begin{array}{l}\text { Age at baseline (months) } \\
\text { Mean (SD) }\end{array}$ & $5.67(3.24)$ & $6.33(2.71)$ & $6.00(2.98)$ & $7.84(3.25)$ \\
Min—Max & $1.8-12.8$ & $1.2-11.6$ & $1.2-12.8$ & $2.6-14.2$
\end{tabular}

$\begin{array}{lllll}\text { Sex (\%) } & & & & \\ \text { Female } & 28.6 & 25.0 & 26.8 & 45.1 \\ \text { Male } & 71.4 & 75.0 & 73.2 & 54.9\end{array}$

$\begin{array}{lrrrr}\text { Race (\%) } & & & & \\ \text { Asian } & 5.7 & 2.8 & 4.2 & 0.0 \\ \text { Black } & 2.9 & 0.0 & 1.4 & 0.0 \\ \text { Caucasian/White } & 88.6 & 88.9 & 88.7 & 92.2 \\ \begin{array}{l}\text { Combination of } \\ \text { above/other }\end{array} & 2.9 & 8.3 & 5.6 & 7.8\end{array}$

$\begin{array}{lllll}\text { Mode of delivery (\%) } & & & & \\ \text { Caesarean section } & 20.0 & 41.7 & 31.0 & 13.7 \\ \text { Vaginal } & 80.0 & 58.3 & 69.0 & 86.3\end{array}$

Country of residence (\%)

$\begin{array}{lrrrr}\text { Belgium } & 17.1 & 13.9 & 15.5 & 0.0 \\ \text { United Kingdom } & 60.0 & 69.4 & 64.8 & 29.4 \\ \text { Italy } & 17.1 & 13.9 & 15.5 & 11.8 \\ \text { Sweden } & 5.7 & 2.8 & 4.2 & 58.8\end{array}$

CMA, cow's milk allergy. Healthy subjects: healthy breastfed reference group. $\mathrm{N}$ is number of subjects. Denominator for $\%$ is number of subjects in the treatment group with non-missing data.

treatment arms were well balanced with respect to baseline demographics-except for the mode of delivery, which was twice as high in the control group compared with that in the test group (Caesarean section $41.7 \%$ and $20.0 \%$, respectively; Table 2). Most subjects were Caucasian and $60 \%$ were recruited in the UK, whereas Sweden contributed most infants in the HBR group. Most CMA subjects suffered from the following symptoms associated with cow's milk protein ingestion: frequent regurgitation or vomiting (72\%), persistent distress or colic (70\%), eczema (52\%), a change in behavior such as irritability or crying (44\%), soft stool constipation (41\%), and faltering growth (34\%; Table 3). GI, skin, respiratory, and other symptoms were well balanced at baseline. GI symptoms were the predominant complaint in $90.1 \%$ of the subjects, whereas the remainder suffered predominantly from skin symptoms. Most infants in test $(29 \%, 46 \%)$ and control groups $(36 \%, 53 \%)$ were already on hydrolysate formula or AAF, respectively, at study entry. The average amount of study formula intake during 
Table 3. Medical history of presenting complaints of subjects in the randomized arms at study baseline

\begin{tabular}{|c|c|c|c|}
\hline Medical history of presenting complaints as examined by clinician, $N(\%)$ & Test $(N=35)$ & Control $(N=36)$ & Total $(N=71)$ \\
\hline \multicolumn{4}{|l|}{ Overall symptoms } \\
\hline A change in behavior such as irritability or crying & $12(34.3 \%)$ & $19(52.8 \%)$ & $31(43.7 \%)$ \\
\hline \multicolumn{4}{|l|}{ Gastrointestinal symptoms } \\
\hline Frequent regurgitation or vomiting related to cow's milk protein & $23(65.7 \%)$ & $29(80.6 \%)$ & $52(72.3 \%)$ \\
\hline Soft stool constipation (with/without perianal rash due to infection) & $12(34.3 \%)$ & $17(47.2 \%)$ & $29(40.8 \%)$ \\
\hline Faltering growth after the dietary inclusion of cow's milk protein & $13(37.1 \%)$ & $11(30.6 \%)$ & $24(33.8 \%)$ \\
\hline Extended periods of diarrhea with a negative stool examination & $8(22.9 \%)$ & $9(25.0 \%)$ & $17(23.9 \%)$ \\
\hline Blood in stool & $10(28.6 \%)$ & $5(13.9 \%)$ & $15(21.1 \%)$ \\
\hline Endoscopically confirmed eosinophilic enteropathy & 0 & 0 & 0 \\
\hline Urticaria & $3(8.6 \%)$ & $4(11.1 \%)$ & 7 (9.9\%) \\
\hline \multicolumn{4}{|l|}{ Respiratory symptoms } \\
\hline Sneezing/nasal congestion & $9(25.7 \%)$ & $12(33.3 \%)$ & $21(29.6 \%)$ \\
\hline Wheezing & $5(14.3 \%)$ & $5(13.9 \%)$ & $10(14.1 \%)$ \\
\hline Conjunctivitis & $1(2.9 \%)$ & $3(8.3 \%)$ & $4(5.6 \%)$ \\
\hline Dyspnea & $1(2.9 \%)$ & $1(2.8 \%)$ & $2(2.8 \%)$ \\
\hline Stridor & $1(2.9 \%)$ & $1(2.8 \%)$ & $2(2.8 \%)$ \\
\hline Dysphonia & 0 & 0 & 0 \\
\hline \multicolumn{4}{|l|}{ Predominant complaint/stratification factor } \\
\hline Skin symptoms & $4(11.4 \%)$ & $3(8.3 \%)$ & 7 (9.9\%) \\
\hline Gastrointestinal symptoms & 31 (88.6\%) & $33(91.7 \%)$ & $64(90.1 \%)$ \\
\hline
\end{tabular}

the study did not differ between study groups. In week 1 , the mean daily intake $( \pm$ SD) was $602( \pm 247)$ and $627( \pm 205) \mathrm{ml}$ in test and control group, respectively $(P=0.646)$. The mean daily intake for test and control groups were $629( \pm 213)$ and $660( \pm 238) \mathrm{ml}(P=0.603)$ and $652( \pm 176)$ and $639( \pm 212) \mathrm{ml}$ $(P=0.797)$ in weeks 4 and 8 , respectively. Nature and frequency of solid foods consumed during the study were well balanced between test and control groups as well as between the HBR group and both CMA groups at week 8 (data not show).

The primary outcome showed statistically significant differences $(P<0.001)$ between test and control groups at week 8 in the fecal composition of bifidobacteria and ER/CC (Figure 2). In subjects given AAF including synbiotics, the median percentages of bifidobacteria were higher at week 8 compared with those in the control group (35.4\% vs. 9.7\%, respectively), whereas the median percentages of adult-like ER/CC were lower $(9.5 \%$ vs. $24.2 \%$, respectively). The differences between test and control arms were statistically significant for bifidobacteria $(+20.937 \%$ (95\% confidence intervals $10.14,31.74) ; P<0.001)$ and $\mathrm{ER} / \mathrm{CC}(-14.115 \%$ $(-22.21,-6.02) ; P<0.001)$. At week 8 , the median percentages of bifidobacteria and ER/CC of the test group were $35.4 \%$ and $9.5 \%$, respectively, which approximated the levels in the HBR (55\% and 6.5\%) more so than the levels in the control group ( $9.7 \%$ and $24.2 \%$ ). The sensitivity analyses considered intake of proton pump inhibitors or $\mathrm{H} 2$ antagonists as potential confounder for bifidobacteria analyses and antibiotic use and sex as potential intervention effect modifiers. Including intake of proton pump inhibitors or $\mathrm{H} 2$ 

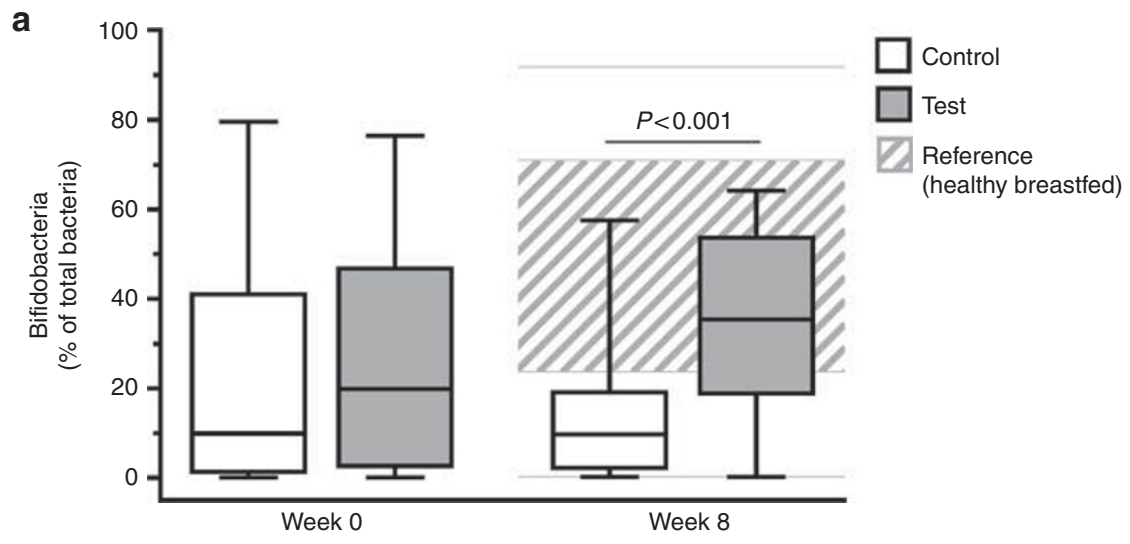

b

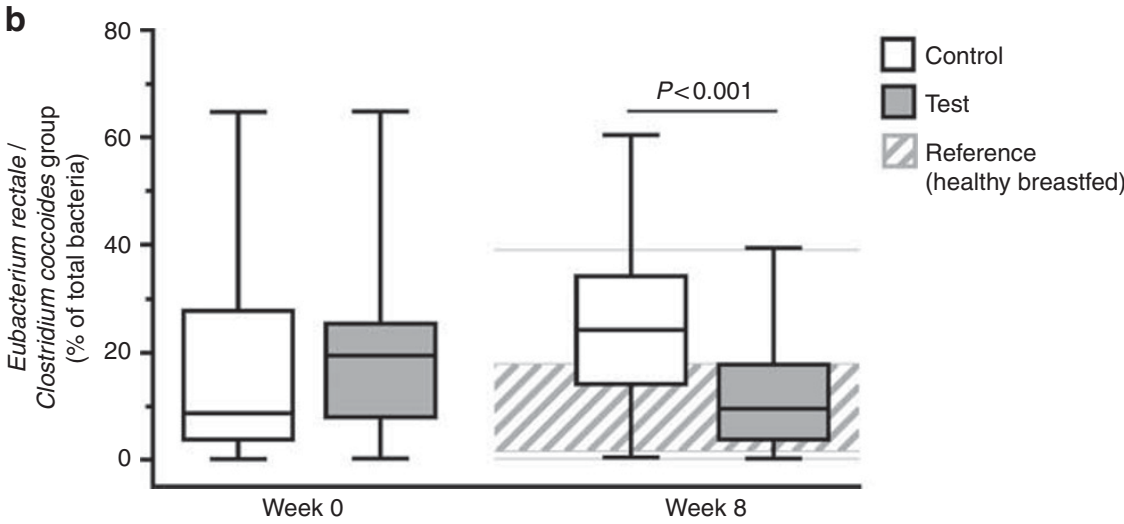

Figure 2. Percentages of bifidobacteria (a) and adult-like $E R / C C$ (b) at weeks 0 and 8 in subjects given test formula or control formula (ITT). The gray shaded area represents the sample 25th to 75th percentile of the healthy reference group (healthy, breastfed subjects), and the gray horizontal lines represent the minimum and maximum values of the healthy reference group. $P$ values are based on ANCOVA comparing test vs. control groups with week 8 values as outcome, stratification factor (skin or gastrointestinal symptoms) and imputed baseline values as covariate and intervention as fixed effect, respectively.

antagonists as an additional covariate into the ANCOVA model, however, did not change the outcome of the primary parameters bifidobacteria $(P<0.001)$ and ER/CC $(P<0.001)$ at week 8. A subgroup analysis on subjects who did not take any systemic antibiotics $(n=47)$ showed that differences between test and control groups were statistically significant for both bifidobacteria $(P<0.001)$ and ER/CC $(P<0.001$; Supplementary Figure S1 online). In addition, the differences between the test and control groups at week 8 were also statistically significant in both males $(n=41)$ and females $(n=15)$ for bifidobacteria $(P=0.037$ and $P<0.001$, respectively $)$ and ER/CC $(P=0.047$ and $P=0.032$, respectively; Supplementary Figure S1). The sensitivity analyses showed that all other predetermined covariablesincluding age at baseline, mode of delivery, introduction of weaning foods, total duration of breastfeeding, study site, and country-did not significantly influence primary outcome.

Stool frequency score was lower in the test group than in the control group $(1.88 \pm 0.19$ vs. $1.98 \pm 0.15 ; P=0.015)$; however, all other measures of stool characteristics were not statistically significantly different between groups at week 8 (data not shown).
In exploratory analyses of clinical outcomes, no statistically significant differences were observed at week 8 . Figure 3 shows clinical symptoms reported at weeks $0,1,4$, and 8 . GI symptoms were predominant in the study population. Reported scores for GI and general symptoms were, compared with other assessed outcomes, relatively high at baseline (2.0-2.5) and decreased over time (Figure 3). In contrast, reported scores for skin symptoms were relatively mild at baseline (generally below 1.5), and showed no statistically significant changes over 8 weeks. SCORAD decreased between weeks 0 and 8 , from $12.83 \pm 18.84$ to $9.63 \pm 12.45$ in the test group and from $14.43 \pm 19.74$ to $7.06 \pm 10.01$ in the control group.

Overall, there were no differences in the number of subjects reporting adverse events between test and control groups during the first 8 weeks (Table 4). The total number of concomitant medications taken was lower in subjects given test formula (82) compared with those given control (111) formula, although the number of subjects $(\mathrm{N}=21$ vs $\mathrm{N}=28$, respectively) was not statistically significant between arms. Further evaluation showed a significantly lower percentage of subjects in the test group needed medication related to the subcategory designated "systemic anti-infectives" (subgroup 

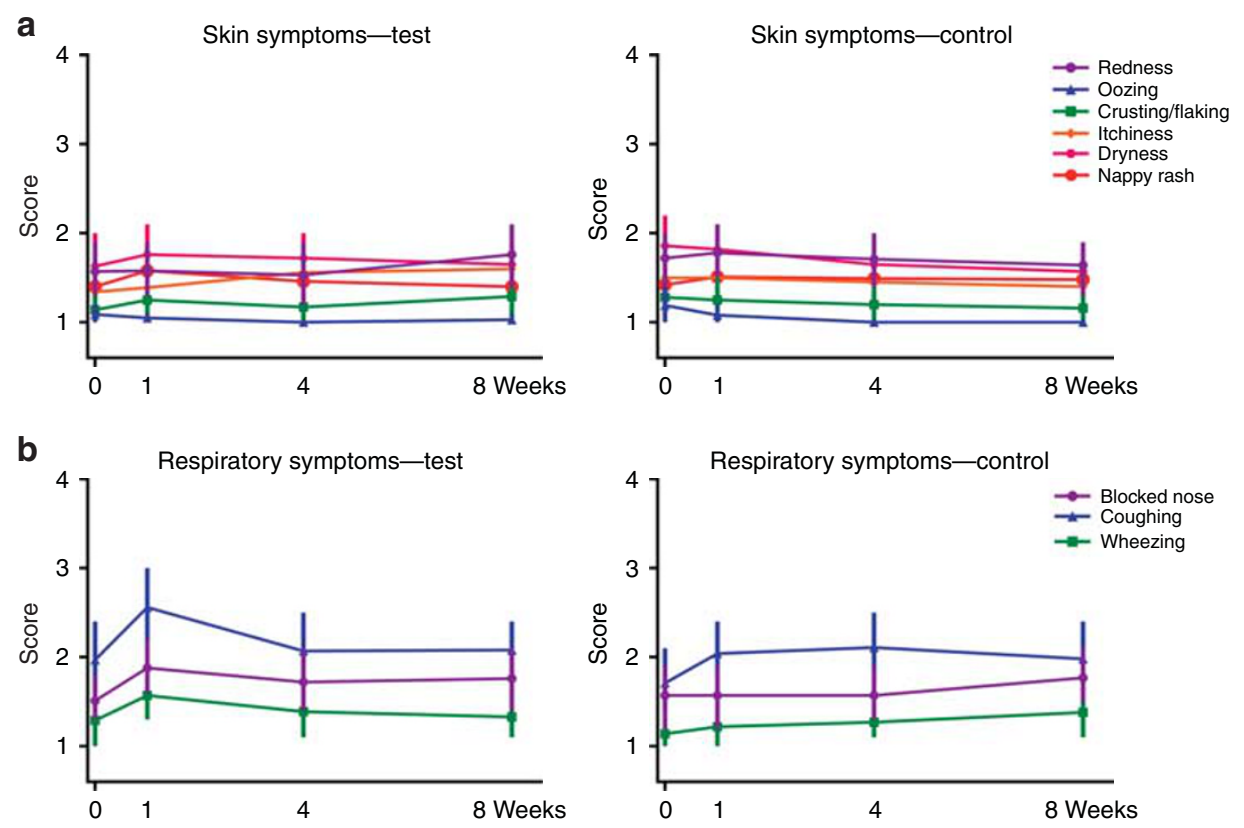

C General and gastrointestinal symptoms-test
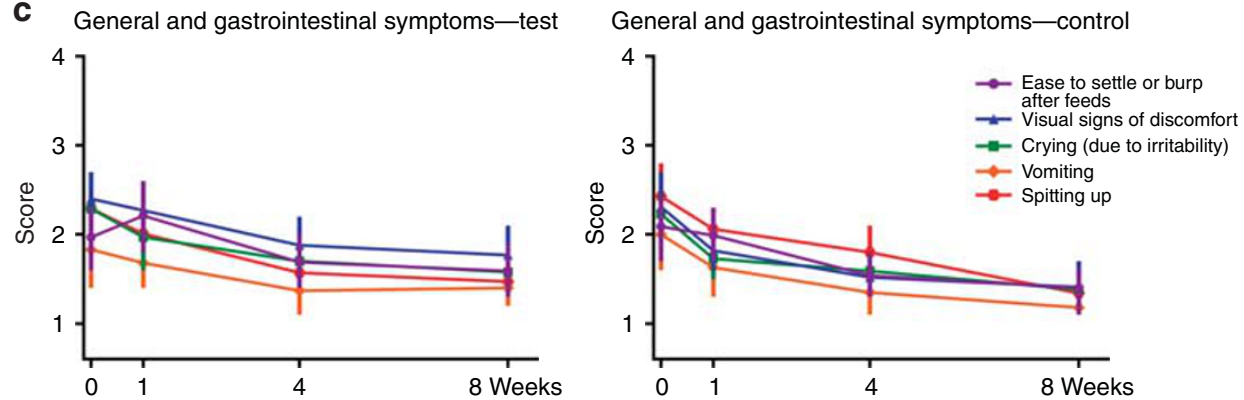

Figure 3. Parent-reported, clinician-evaluated symptoms at weeks $0,1,4$, and 8 assessed on a 4-point rating scale specific for each symptom. Skin symptoms (redness, oozing, crusting, itchiness, dryness, and nappy rash) were rated as 1: none, 2: slight, 3: some, and 4: a lot. Respiratory symptoms blocked nose and wheezing rated as 1: none, 2: mild, 3: moderate, and 4: severe, and coughing was rated as 1: none, 2: one to two times/day, 3: three to five times/day, and 4: more than five times/day. General and gastrointestinal symptoms: vomiting was rated as 1: none, 2: one to two times/ day, 3: three to four days/day, and 4: more than four times/day; spitting-up as 1: none, 2: after some feeds, 3: after all feeds, and 4: between and after feeds; gas/wind as 1: none; 2: slight; 3: some; and 4: a lot; sleep pattern last night as 1: normal, 2: awake once, 3: awake two to three times, and 4: awake more than three times; ease of settling or burping after feeds as 1: no problem at all, 2: slight difficulty, 3: some difficulty, and 4: very difficult; visual signs of discomfort (e.g., back arching) as 1: none, 2: slight, 3: some, and 4: a lot; and crying (because of irritability) as 1: none, 2: up to $1 \mathrm{~h}, 3: 1-3 \mathrm{~h}$, and 4 : more than $3 \mathrm{~h}$. Data are shown as mean values $\pm 95 \%$ confidence interval limits.

according to the Anatomical Therapeutic Chemical coding system, which includes antibacterials for systemic use and vaccines) compared with that in the control group (8.6\% vs. $34.4 \%$, respectively; $P=0.018$ ).

Growth parameters were within the expected ranges for age and median $Z$-scores for both groups were within $1 \mathrm{SD}$ of the mean (data not shown).

\section{DISCUSSION}

The primary objective of modifying gut microbiota using an AAF including a blend of prebiotic fructo-oligosaccharides and the probiotic strain $B$. breve $\mathrm{M}-16 \mathrm{~V}$ for 8 weeks in subjects with suspected non-IgE CMA was achieved. Percentages of bifidobacteria were higher and adult-like ER/CC lower among infants given the AAF with these specific synbiotics compared with those given the AAF without synbiotics.

This study was primarily designed to investigate whether the synbiotic ingredients can improve the gut microbiota in infants with non-IgE CMA to achieve a microbial composition close to that seen in healthy, breastfed infants. Previous studies showing the effects of breast milk on gut microbiota $(33,34)$ helped to guide the development of this AAF, which contains a specific blend of prebiotics and probiotics. $B$. breve is a bacterial species found in human milk and the gut of healthy infants $(33,35)$, and in preclinical models $B$. breve $\mathrm{M}-16 \mathrm{~V}$ was identified as the most potent anti-allergic probiotic strain tested (36). Another model of established food allergy showed potential immunomodulatory benefits of dietary intervention with a synbiotic combination of olifructose and inuline (short- and long-chain fructo-oligosaccharides) and 


\section{Articles | Candy et al.}

Table 4. Adverse events and use of concomitant medications in test and control groups

\begin{tabular}{ccc}
\hline Test & Control & $P$ value \\
$(N=35)$ & $(N=36)$ & $\begin{array}{c}\text { (Fisher's } \\
\text { exact test) }\end{array}$ \\
& & \\
\hline
\end{tabular}

Adverse events, $N$ (\%)

Overall

Any adverse event

$20(57.1 \%) 23(65.7 \%)$

0.624

Severity

Mild

$15(42.9 \%) 15(42.9 \%)$

Moderate

$4(11.4 \%) \quad 7(20.0 \%)$

Severe $^{\text {a }}$

$1(2.9 \%) \quad 1(2.9 \%)$

Preferred term description ${ }^{\mathrm{b}}$

Gastrointestinal disorders

$11(31.4 \%) 13(37.1 \%)$

0.802

Infections and infestations

$10(28.6 \%) 12(34.3 \%)$

0.797

Concomitant medication, $N$ (\%)

Overall

$\begin{array}{llll}\text { Any concomitant medication } & 21(60.0 \%) & 28(80.0 \%) & 0.117\end{array}$

Subcategoryc

Anti-infectives for systemic use $3(8.6 \%) \quad 12(34.4 \%) \quad 0.018$

aReported severe adverse events were feeding disorder of infancy or early childhood (test group) and bronchiolitis and feeding disorder of infancy or early childhood (control group).

${ }^{\text {b}}$ The two most frequently reported preferred terms of adverse event are shown. ${ }^{c}$ Only categories (of total nine categories) with a statistically significant difference $(P<0.05)$ are shown.

B. breve M-16V (21). The current study showed that microbial composition of infants with suspected non-IgE CMA who received the test formula was closer to the profile of the HBR group than those infants receiving control formula. On the basis of previous studies, we hypothesized that modifying the gut microbiota with these specific synbiotics may improve clinical symptoms associated with gut microbiota dysbiosis and dysregulated immune reactions in infants with CMA $(37,38)$. Overall, exploratory GI and general symptoms improved over time, but were not statistically significantly different between test and control groups at week 8 . This trial was not primarily designed or powered to show differences in clinical outcomes between groups, and it is important to note that the majority of subjects were already receiving a hydrolyzed formula or AAF at study entry, which confounds interpretation of the exploratory data analysis.

This randomized trial has several limitations. The trial was designed to evaluate the effects of AAF with synbiotics exclusively in subjects with non-IgE CMA. There is no standard test for the precise diagnosis of non-IgE allergy, raising the possibility that subjects with other conditions, for example, other (food) allergy presentations, could dilute the trial population. The possibility of erroneous entry into the trial was addressed by using a robust diagnostic work-up (Table 1) collectively designed by a multidisciplinary team of clinicians, comprising pediatric gastroenterology, allergy, and immunology, and was based on careful symptom assessment by the investigators, with specific IgE testing and skin-prick testing (if assessed) to exclude any IgE-mediated CMA. Additional research is warranted to define precise biomarkers for this allergic phenotype.

Consistent with scientific methodology, it is essential to ensure that patients meet diagnostic criteria for eligibility and undergo a double-blind placebo-controlled challenge to confirm symptomatology to cow's milk protein. Furthermore, as seen in clinical practice, determining disease resolution requires re-introduction of the food-by-food challenge or introduction at home. In the present trial, it was not mandatory for study subjects to have a food challenge to confirm CMA diagnosis, although this would have been ideal for the interpretation of the clinical outcomes. However, overall, in the context of our understanding of the many influences on the gut microbiota, the investigators do not believe that this specific limitation of the study would have a meaningful influence on the primary outcome.

One of the factors associated with microbiota development is the mode of delivery (i.e., caesarean or vaginal delivery) of an infant. In our study, twice as much caesarean-delivered infants were randomized to the control group compared with test group ( $42 \%$ and $20 \%$, respectively) and could, therefore, potentially influence the primary outcome. However, our statistical analyses showed that this factor did not influence current study outcome.

Furthermore, there is no recognized standard profile for the composition of a healthy microbiota during the dietary diversification period in early life. The trial design partly addresses this issue by using an HBR group to allow agematched comparison of data; however, it is important to recognize that the reference population is not identical to the randomized groups, for example, with respect to predominant country of origin, mode of delivery (14\% caesarean sectionborn vs. $20 \%$ and $42 \%$ in test and control group, respectively), general health status, allergic symptoms, and dietary management. The authors feel that despite these limitations, this HBR group is a good representation of a healthy microbiota to function as reference. The duration of formula administration and length of follow-up mean there is only limited scope for this trial to assess longer-term changes in gut microbiota.

This randomized controlled trial adds to the evidence base for prebiotics and probiotics in the alteration of infant microbiota and potentially the dietary management of CMA (22-25,27,39-42). However, caution is required in making comparisons between studies, which had different trial entry criteria and tested a range of dietary management strategies. The test formula in this trial was a hypoallergenic, nutritionally complete AAF with a specific composition of prebiotics and probiotics, not derived from cow's milk ingredients. The formula composition was similar to the one tested by Harvey et al. (26), but excluded the pectinderived acidic oligosaccharides. Data with this formula cannot be extrapolated to other types of formula, e.g., eHF, or formulae containing different types of prebiotics or alternative strains of probiotics.

Safety concerns have been expressed with other infant formulae containing different probiotics $(43,44)$, and it is 


\section{Synbiotic improves CMA-infant microbiota}

important that safety is established in clinical trials for each specific formula in an appropriate population (45). The synbiotic-supplemented AAF in this trial was shown to be safe in terms of adverse events, use of concomitant medications, and achievement of growth targets (26). Several studies have shown that $B$. breve $\mathrm{M}-16 \mathrm{~V}$ is safe in infants, including preterm neonates $(26,27,46)$. Although the addition of these specific synbiotics to AAF improves the composition of the gut microbiota so that it more closely resembles the composition observed in breastfed individuals, the results of this trial do not change the recommendation that infants with CMA should be fed with human breast milk if possible.

Although these results are specific to subjects with non-IgEmediated CMA, Burks et al. (28) showed that an AAF, including ingredients from the current synbiotic blend, was safe in patients with IgE and non-IgE-mediated CMA. An ongoing clinical study (Netherlands Trial Register NTR3725) includes infants with confirmed IgE-mediated CMA, randomly allocated to receive AAF with or without synbiotics for 12 months, and will assess cow's milk tolerance acquisition over 24 months. The ongoing and reported trial will inform future studies primarily focusing at clinical outcomes in the specific CMA populations.

On the basis of the data showing significant effects on the composition of gut microbiota that extend beyond an increase in bifidobacteria, we conclude that the AAF including the specific synbiotics of fructo-oligosaccharides and $B$. breve $\mathrm{M}-16 \mathrm{~V}$ used in this trial was equally well tolerated as AAF without synbiotics, suitable for dietary management, and supports microbiota development of infants with suspected non-IgE-mediated CMA.

\section{SUPPLEMENTARY MATERIAL}

Supplementary material is linked to the online version of the paper at http://www.nature.com/pr

\section{ACKNOWLEDGMENTS}

We thank Rob Slump of the department of Gut Biology and Microbiology of Nutricia Research, The Netherlands, for the analysis of the gut microbiota data. We also thank the Clinical Study, Analytical Sciences, and Data sciences teams of Nutricia Research, The Netherlands, and medical writer Tim Kelly for suggestions and critical review of the manuscript.

\section{STATEMENT OF FINANCIAL SUPPORT}

This study was funded by Nutricia Research B.V.

\section{ASSIGN INVESTIGATORS TO THIS STUDY GROUP}

Francoise Smets, U.C.L. Saint-Luc, Brussels, Belgium; Sandra Mullier, HUDERF, Brussels, Belgium; Lee Noimark, Barts/Royal Hospital, London, United Kingdom; Antonella Muraro, University Hospital of Padova, Padova, Italy.

Disclosure: None of the authors have financial ties to products in the study or potential conflict of interest. Authors affiliated to Nutricia Research receive salary, which is not related to study outcome.

\section{REFERENCES}

1. Vandenplas Y, De Greef E, Devreker T. Treatment of cow's milk protein allergy. Pediatr Gastroenterol Hepatol Nutr 2014;17:1-5.
2. Nwaru BI, Hickstein L, Panesar SS, et al. Prevalence of common food allergies in Europe: a systematic review and meta-analysis. Allergy 2014;69:992-1007.

3. Fiocchi A, Brozek J, Schunemann H, et al. World Allergy Organization (WAO) Diagnosis and Rationale for Action against Cow's Milk Allergy (DRACMA) Guidelines. Pediatr Allergy Immunol 2010;21 (Suppl 21): $1-125$.

4. Schoemaker AA, Sprikkelman AB, Grimshaw KE, et al. Incidence and natural history of challenge-proven cow's milk allergy in European children-EuroPrevall birth cohort. Allergy 2015;70:963-72.

5. Luyt D, Ball H, Makwana N, et al. BSACI guideline for the diagnosis and management of cow's milk allergy. Clin Exp Allergy 2014;44:642-72.

6. Venter C, Brown T, Shah N, Walsh J, Fox AT. Diagnosis and management of non-IgE-mediated cow's milk allergy in infancy - a UK primary care practical guide. Clin Transl Allergy 2013;3:23.

7. Anagnostou K, Meyer R, Fox A, Shah N. The rapidly changing world of food allergy in children. F1000Prime Rep 2015;7:35.

8. Koletzko S, Niggemann B, Arato A, et al. Diagnostic approach and management of cow's-milk protein allergy in infants and children: ESPGHAN GI Committee practical guidelines. J Pediatr Gastroenterol Nutr 2012;55:221-9.

9. Gensollen T, Iyer SS, Kasper DL, Blumberg RS. How colonization by microbiota in early life shapes the immune system. Science 2016;352: 539-44.

10. McCoy KD, Koller Y. New developments providing mechanistic insight into the impact of the microbiota on allergic disease. Clin Immunol 2015;159:170-6.

11. West CE, Jenmalm MC, Prescott SL. The gut microbiota and its role in the development of allergic disease: a wider perspective. Clin Exp Allergy 2015;45:43-53.

12. Bisgaard $\mathrm{H}, \mathrm{Li} \mathrm{N}$, Bonnelykke $\mathrm{K}$, et al. Reduced diversity of the intestinal microbiota during infancy is associated with increased risk of allergic disease at school age. J Allergy Clin Immunol 2011;128:646-52 e1-5.

13. Bokulich NA, Chung J, Battaglia T, et al. Antibiotics, birth mode, and diet shape microbiome maturation during early life. Sci Transl Med 2016;8: $343 \mathrm{ra} 82$.

14. West CE, Ryden P, Lundin D, Engstrand L, Tulic MK, Prescott SL. Gut microbiome and innate immune response patterns in IgE-associated eczema. Clin Exp Allergy 2015;45:1419-29.

15. Berni Canani R, Sangwan N, Stefka AT, et al. Lactobacillus rhamnosus GG-supplemented formula expands butyrate-producing bacterial strains in food allergic infants. ISME J 2016;10:742-50.

16. Bunyavanich S, Shen N, Grishin A, et al. Early-life gut microbiome composition and milk allergy resolution. J Allergy Clin Immunol. 2016;138:1122-30.

17. Moro GE, Mosca F, Miniello V, et al. Effects of a new mixture of prebiotics on faecal flora and stools in term infants. Acta Paediatr Suppl 2003;91:77-9.

18. Sierra C, Bernal MJ, Blasco J, et al. Prebiotic effect during the first year of life in healthy infants fed formula containing GOS as the only prebiotic: a multicentre, randomised, double-blind and placebo-controlled trial. Eur J Nutr 2015;54:89-99.

19. Veereman-Wauters G, Staelens S, Van de Broek H, et al. Physiological and bifidogenic effects of prebiotic supplements in infant formulae. J Pediatr Gastroenterol Nutr 2011;52:763-1.

20. Schouten B, van Esch BC, Hofman GA, et al. Cow milk allergy symptoms are reduced in mice fed dietary synbiotics during oral sensitization with whey. J Nutr 2009;139:1398-403.

21. van Esch BCAM, Abbring S, Diks MAP, et al. Post-sensitization administration of non-digestible oligosaccharides and Bifidobacterium breve M-16 V reduces allergic symptoms in mice. Immun Inflamm Dis 2016;4:155-65.

22. Berni Canani R, Nocerino R, Terrin G, et al. Effect of Lactobacillus GG on tolerance acquisition in infants with cow's milk allergy: a randomized trial. J Allergy Clin Immunol 2012;129:580-2 582 e1-5.

23. Dupont C, Hol J, Nieuwenhuis EE. An extensively hydrolysed caseinbased formula for infants with cows' milk protein allergy: tolerance/hypoallergenicity and growth catch-up. Br J Nutr 2015;113:1102-2. 


\section{Articles | Candy et al.}

24. Muraro A, Hoekstra MO, Meijer Y, et al. Extensively hydrolysed casein formula supplemented with Lactobacillus rhamnosus GG maintains hypoallergenic status: randomised double-blind, placebo-controlled crossover trial. BMJ Open 2012;2:e000637.

25. Vandenplas Y, Steenhout P, Planoudis Y, Grathwohl D, Althera Study G. Treating cow's milk protein allergy: a double-blind randomized trial comparing two extensively hydrolysed formulas with probiotics. Acta Paediatr 2013;102:990-8.

26. Harvey BM, Langford JE, Harthoorn LF, et al. Effects on growth and tolerance and hypoallergenicity of an amino acid-based formula with synbiotics. Pediatr Res 2014;75:343-51.

27. Burks AW, Harthoorn LF, Van Ampting MT, et al. Synbioticssupplemented amino acid-based formula supports adequate growth in cow's milk allergic infants. Pediatr Allergy Immunol 2015;26:316-22.

28. Dupont C. Diagnosis of cow's milk allergy in children: determining the gold standard? Expert Rev Clin Immunol 2014;10:257-67.

29. van der Aa LB, Heymans HS, van Aalderen WM, et al. Effect of a new synbiotic mixture on atopic dermatitis in infants: a randomizedcontrolled trial. Clin Exp Allergy 2010;40:795-804.

30. Martin R, Makino H, Cetinyurek Yavuz A, et al. Early-life events, including mode of delivery and type of feeding, siblings and gender, shape the developing gut microbiota. PLoS ONE 2016;11:e0158498.

31. Cheng J, Ringel-Kulka T, Heikamp-de Jong I, et al. Discordant temporal development of bacterial phyla and the emergence of core in the fecal microbiota of young children. ISME J 2016;10:1002-4.

32. Severity scoring of atopic dermatitis: the SCORAD index. Consensus Report of the European Task Force on Atopic Dermatitis. Dermatology 1993;186:23-31.

33. Martin R, Jimenez E, Heilig $H$, et al. Isolation of bifidobacteria from breast milk and assessment of the bifidobacterial population by PCRdenaturing gradient gel electrophoresis and quantitative real-time PCR. Appl Environ Microbiol 2009;75:965-9.

34. Praveen P, Jordan F, Priami C, Morine MJ. The role of breast-feeding in infant immune system: a systems perspective on the intestinal microbiome. Microbiome 2015;3:41.

35. Enomoto T, Sowa M, Nishimori K, et al. Effects of bifidobacterial supplementation to pregnant women and infants in the prevention of allergy development in infants and on fecal microbiota. Allergol Int 2014;63:575-85.

36. Hougee S, Vriesema AJ, Wijering SC, et al. Oral treatment with probiotics reduces allergic symptoms in ovalbumin-sensitized mice: a bacterial strain comparative study. Int Arch Allergy Immunol 2010;151:107-7.
37. Jyonouchi H. Non-IgE mediated food allergy - update of recent progress in mucosal immunity. Inflamm Allergy Drug Targets 2012;11:382-96.

38. Walker WA, Iyengar RS. Breast milk, microbiota, and intestinal immune homeostasis. Pediatr Res 2015;77:220-8.

39. Ahanchian H, Nouri Z, Jafari SA, et al. Synbiotics in children with cow's milk allergy: a randomized controlled trial. Iran J Pediatr 2014;24:29-34.

40. Hol J, van Leer EH, Elink Schuurman BE, et al. The acquisition of tolerance toward cow's milk through probiotic supplementation: a randomized, controlled trial. J Allergy Clin Immunol 2008;121:1448-54.

41. Ivakhnenko ES, Nian'kovskii SL. [Effect of probiotics on the dynamics of gastrointestinal symptoms of food allergy to cow's milk protein in infants]. Georgian Med News 2013;219:46-52.

42. Ivakhnenko O, Niankovskyy S. Clinical effectiveness of probiotics in complex treatment of infants with cow's milk allergy. Georgian Med News 2013;216:39-45.

43. Chiang WC, Huang CH, Llanora GV, et al. Anaphylaxis to cow's milk formula containing short-chain galacto-oligosaccharide. J Allergy Clin Immunol 2012;130:1361-7.

44. Martin-Munoz MF, Fortuni M, Caminoa M, Belver T, Quirce S, Caballero T. Anaphylactic reaction to probiotics. Cow's milk and hen's egg allergens in probiotic compounds. Pediatr Allergy Immunol 2012;23:778-84.

45. van den Nieuwboer M, Claassen E, Morelli L, Guarner F, Brummer RJ. Probiotic and synbiotic safety in infants under two years of age. Benef Microbes 2014;5:45-60.

46. Patole S, Keil AD, Chang A, et al. Effect of Bifidobacterium breve M-16V supplementation on fecal bifidobacteria in preterm neonates-a randomised double blind placebo controlled trial. PLoS ONE 2014;9:e89511.

(i) This work is licensed under a Creative Commons Attribution 4.0 International License. The images or other third party material in this article are included in the article's Creative Commons license, unless indicated otherwise in the credit line; if the material is not included under the Creative Commons license, users will need to obtain permission from the license holder to reproduce the material. To view a copy of this license, visit http://creativecommons.org/licenses/by/4.0/

(C) The Author(s) (2018) 